\title{
Medição da densidade do óleo: uma discussão sobre sua otimização e diminuição dos custos via incerteza relativa da medição
}

(Oil density measurement: a discussion about the optimization and decreasing of the costs via relative uncertainty of the measurement)

\author{
V.L.B. de Jesus ${ }^{1}$ e D.A. Palma \\ Centro Federal de Educação Tecnológia de Química de Nilópolis, Nilópolis, RJ, Brasil \\ Recebido em 7/1/2008; Revisado em 18/2/2008; Aceito em 30/3/2008; Publicado em 2/8/2008
}

No presente trabalho, faz-se uma análise metrológica das incertezas associadas às medições da densidade de óleo de cozinha por dois métodos distintos, a saber, o de medição direta (razão massa-volume) e o método de tubo em U. A idéia é ajudar o professor na montagem de experimentos de baixo custo. Em relação ao método de medição utilizando um tubo em U, melhorias são propostas com a substituição do tubo de vidro por um tubo transparente flexível e de baixo custo. Os autores definem o fator de vantagem de um experimento, $F_{v}$, como sendo o produto da incerteza da grandeza física $(\delta)$ pelo seu custo $(C)$, que pode ser utilizado como um critério para decidir o impasse entre a qualidade dos resultados obtidos e o custo de um determinado experimento.

Palavras-chave: metrologia, densidade do óleo, experimento de baixo custo.

In this work one does a metrological analysis of the associated uncertainties to the kitchen oil density measurements using two different methods: the direct measurement (ratio mass-volume) and U tube method. The idea is to help the teacher in the setup of low cost experiments. About the measurement using the U tube method, improvements are proposed substituting the U glass tube for a low cost transparent flexible tube. The authors define the profit factor of an experiment, $F_{v}$, as being the product of the uncertainty in the physical quantity $(\delta)$ by its cost $(C)$. The profit factor can be used as a criterion to decide between the quality of the obtained results and the cost of an experiment.

Keywords: metrology, oil density, inexpensive experiment.

\section{Introdução}

Em meados da década de 80 constatou-se, a partir de diversos trabalhos, uma preocupação por parte dos professores de física nos ensinos fundamental e médio com a questão da experimentação nos laboratórios, seja como fator motivador, ou como complemento do que é ensinado em sala de aula. Neste cenário, o professor de física assume muitas vezes o papel de gestor, sendo responsável pela compra e montagem de equipamentos de cunho didático em espaços físicos adaptados para tal fim e com verbas reduzidas. A idéia é ajudar o professor na montagem de experimentos de baixo custo utilizando uma análise metrológica das incertezas associadas às medições. Será utilizada como exemplo a medição da densidade de óleo de cozinha por dois métodos distintos, a saber, o de medição direta (razão massa-volume) e o método de tubo em U.

A medição da densidade do óleo de cozinha (ou qualquer outro tipo de óleo) pode ser realizada via sua

\footnotetext{
${ }^{1}$ E-mail: vitor@cefeteq.br.

definição padrão, ou seja, a medida de sua massa dividida pelo seu volume. Esta é uma prática comum em nosso laboratório didático de física. A exatidão da medição vai depender do tipo de proveta (instrumento cilíndrico de medida de volume para líquidos que pode ser fabricado em vidro ou plástico, com volumes que normalmente variam entre 5 e $2000 \mathrm{~mL}$ ) e de balança utilizados para as medições do volume e da massa, respectivamente. Um exemplo é mostrado na Fig. 1.

Uma outra maneira de se medir a densidade do óleo é ilustrada no método do tubo em U, normalmente feito de vidro, onde são colocados água destilada e óleo. Depois de atingido o equilíbrio hidrostático, são realizadas as medições das alturas das colunas de óleo e água, tomando como referência o nível inferior da coluna de óleo, conforme mostra a Fig. 2. Utilizando a equivalência de pressão nos níveis inferiores das colunas de água e óleo em cada ramo do tubo em U, pode-se obter uma expressão onde a densidade do óleo é proporcional à razão das alturas medidas para a coluna de água 
e de óleo, respectivamente, vezes a densidade da água $\left(0,9982 \mathrm{~g} / \mathrm{cm}^{3}\right.$ a $\left.20{ }^{\circ} \mathrm{C}\right)$. Observa-se aqui que é de fundamental importância uma boa medição das alturas das colunas de óleo e de água.

O método direto (medições diretas da massa e do volume do óleo) permite uma boa exatidão quando utilizamos uma proveta graduada e uma balança que mede até décimos ou centésimos de grama. Surpreendentemente, pode-se alcançar um resultado com exatidão semelhante, ou até mesmo melhor, quando utilizamos uma mangueira transparente, facilmente adquirida em qualquer loja de ferragens, como um tubo em U flexível que pode variar em seu comprimento, dependendo da exatidão desejada. Certamente, um experimento de baixo custo que ilustra o princípio dos vasos comunicantes, o princípio de pressão dependente da profundidade e ainda permite conseguir uma excelente medição da densidade do óleo de cozinha.

O objetivo deste trabalho é abordar a exatidão destes experimentos, seus custos, e mostrar que estas avaliações metrológicas são necessárias. Estas abordagens são feitas em nossas aulas experimentais da disciplina Física Geral IV - Fluidos e Termodinâmica - oferecida no curso de licenciatura em física do Centro Federal de Educação Tecnológica de Química de Nilópolis/RJ.

\section{Metodologias aplicadas na deter- minação da densidade do óleo de co- zinha}

Existem diversos métodos para determinação da densidade de sólidos e líquidos [1]. Na presente seção faz-se uma breve discussão sobre um método tradicional utilizado em laboratórios didáticos de física e/ou química, o chamado método direto, baseado na determinação direta da massa e volume do óleo [2]. Um outro método muito conhecido é utilizado para se determinar à densidade de substâncias líquidas não miscíveis no qual a densidade de um dos líquidos é conhecida, a saber, o método do tubo em U. Uma melhoria é proposta ao método do tubo em U utilizando material de baixo custo e alta eficácia no que tange a redução da incerteza relativa associada à medição da densidade do óleo.

\subsection{Medição direta da densidade do óleo}

A Fig. 1 ilustra o método direto de medição da densidade do óleo de cozinha. Neste experimento utilizamos uma proveta graduada de $500 \mathrm{~mL}$ e menor divisão de $5 \mathrm{~mL}$. A balança digital para medição da massa tem como último algarismo o centésimo de grama.

Os valores obtidos para a massa, $m$, e volume, $V$, são respectivamente, $(437,72 \pm 0,01) \mathrm{g}$ e $(480 \pm 3) \mathrm{mL}$. O método direto para determinação da densidade do óleo consiste em calcular a razão

$$
\rho=\frac{m}{V} \text {. }
$$

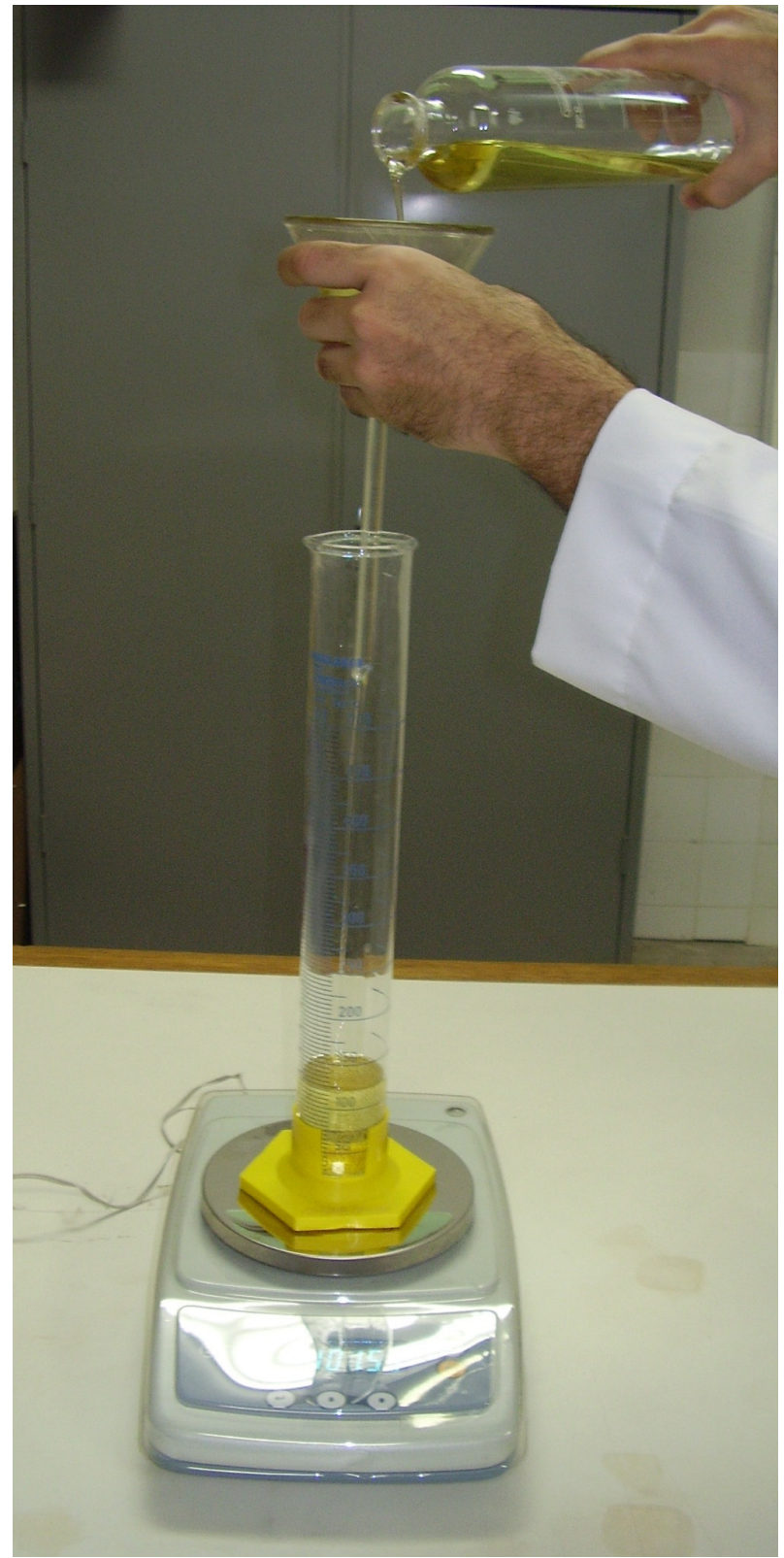

Figura 1 - Medição experimental direta da densidade do óleo de cozinha.

O resultado obtido pela Eq. (1) é o resultado da medição. Este resultado é uma aproximação ou estimativa para o valor verdadeiro da densidade (mensurando). A apresentação de um resultado é completa somente quando acompanhado por uma quantidade que declara a sua incerteza $[3,4]$.

A incerteza combinada a partir do método direto é calculada, até primeira ordem, através da equação [3-5]

$$
\delta \rho^{2}=\left(\frac{\partial \rho}{\partial m} \delta m\right)^{2}+\left(\frac{\partial \rho}{\partial V} \delta V\right)^{2},
$$

onde $\delta m$ é a incerteza do tipo B [3-5] do instrumento de medição da massa (no caso, a balança digital) e $\delta V$ é a incerteza do tipo B do instrumento de medição do volume (no caso, a proveta). Calculando as derivadas 
parciais (também chamados de coeficientes de sensibilidade) chega-se a seguinte expressão para a incerteza da densidade do óleo

$$
\delta \rho^{2}=\left(\frac{1}{V} \delta m\right)^{2}+\left(-\frac{m}{V^{2}} \delta V\right)^{2} .
$$

Dividindo-se a Eq. (3) por $\rho$ obtém-se a incerteza relativa

$$
\left(\frac{\delta \rho}{\rho}\right)^{2}=\left(\frac{\delta m}{m}\right)^{2}+\left(\frac{\delta V}{V}\right)^{2} .
$$

A Eq. (4) nos ajuda no entendimento de que existem três formas básicas de melhorar nosso experimento no sentido de diminuir a incerteza relativa. Uma é diminuir a incerteza associada à medição da massa adquirindo uma balança mais sensível e, portanto, mais onerosa (o que é inviável para muitos estabelecimentos de ensino). Outra seria diminuir a incerteza associada à medição do volume utilizando uma bureta (instrumento cilíndrico de medida de volume para líquidos, de vidro, colocado na vertical com a ajuda de um suporte, contendo uma escala graduada rigorosa. Possui na extremidade inferior uma torneira de precisão para dispensa de volumes rigorosamente conhecidos.). Em geral, buretas possuem volumes máximos reduzidos comparados às provetas. Em nosso caso, a redução da incerteza associada à medição do volume pode não reduzir de forma significativa a incerteza relativa já que o volume e a massa serão também reduzidos (ver Tabela 2). Uma terceira maneira, e com certeza a menos custosa, seria aumentar a massa e, conseqüentemente, o volume de óleo visto que essas quantidades aparecem explicitamente nos denominadores da Eq. (4). Esse exemplo simples ilustra como uma análise metrológica subsidia nas decisões tomadas num laboratório didático, inclusive, na questão de aquisição e manuseio de materiais. Esta análise é válida para qualquer grandeza física baseada na razão entre duas outras grandezas físicas. Os resultados obtidos serão reportados na seção 4 .

\subsection{Medição utilizando o tubo de vidro em U}

A Fig. 2 ilustra um tubo de vidro em U utilizado normalmente para demonstrar a diferença de densidade entre água e óleo, além de mostrar a possibilidade da medição da densidade relativa via os valores das colunas de água e óleo, tendo como referência o nível da superfície de separação entre os dois líquidos. O tubo de vidro em U utilizado tem altura máxima de $10 \mathrm{~cm}$. As medições das alturas das colunas de água e óleo foram, respectivamente, $(7,7 \pm 0,1) \mathrm{cm} \mathrm{e}(8,1 \pm 0,1) \mathrm{cm}$. Foi utilizada uma régua milimetrada e não foi possível atribuir às alturas das colunas de líquido incerteza menor que $0,1 \mathrm{~cm}$, devido a não uniformidade da superfície que separa a água do óleo [5].

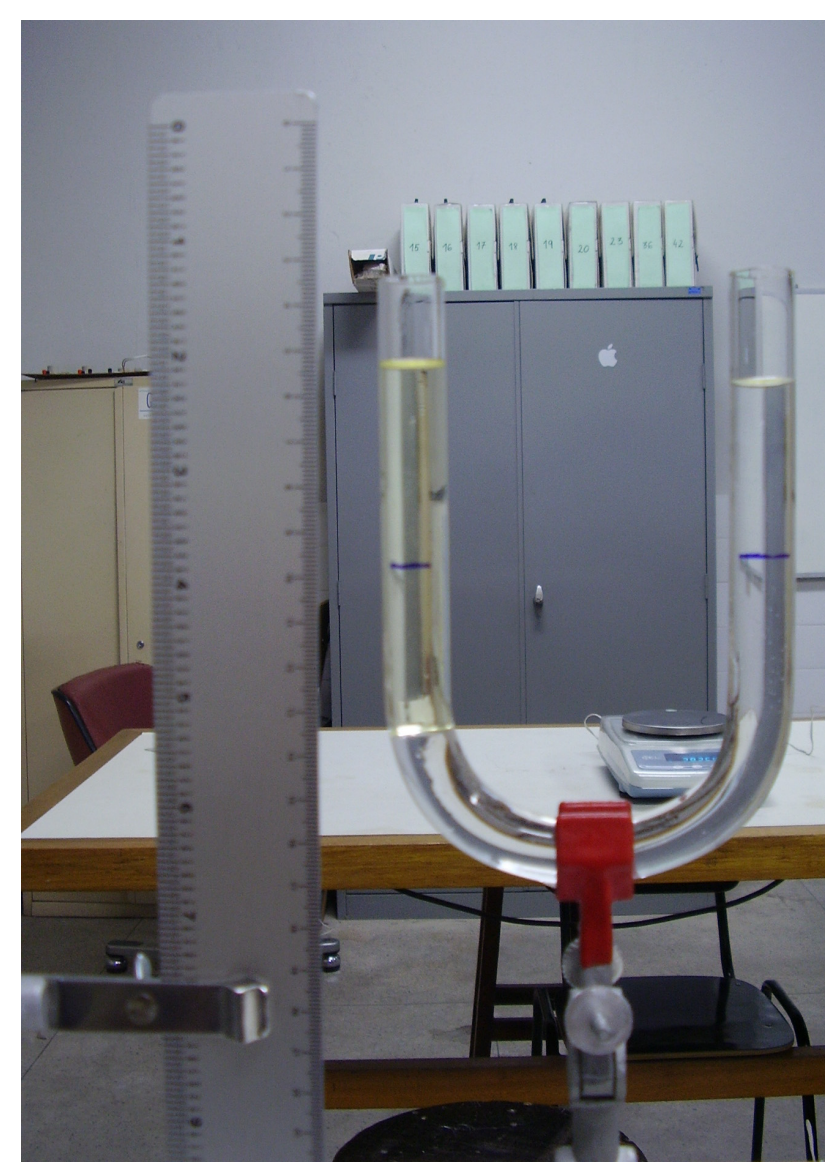

Figura 2 - Medição experimental da densidade do óleo de cozinha utilizando um tubo de vidro em U de altura $10,0 \mathrm{~cm}$.

Utilizando o princípio de Pascal, podemos escrever para as pressões absolutas nos pontos da superfície que separa a água do óleo e seu equivalente do lado oposto como sendo

$$
\rho_{o}=\frac{h_{w}}{h_{o}} \rho_{w}
$$

onde $\rho_{o}$ é a densidade da óleo, $\rho_{w}$ é a densidade da água, $h_{0}$ é a altura da coluna de óleo e $h_{w}$ é a altura da coluna de água. Com o objetivo de expressar a densidade do óleo com sua incerteza associada, ou seja, na forma $\left(\rho_{0}+\delta \rho_{0}\right)$ deve-se combinar as diferentes incertezas associadas às quantidades encontradas na Eq. (5). A incerteza combinada é calculada, até primeira ordem, da seguinte maneira [3-5]

$$
\delta \rho_{0}^{2}=\left(\frac{\partial \rho_{0}}{\partial h_{w}} \delta h_{w}\right)^{2}+\left(\frac{\partial \rho_{0}}{\partial h_{0}} \delta h_{0}\right)^{2}+\left(\frac{\partial \rho_{0}}{\partial \rho_{w}} \delta \rho_{w}\right)^{2} .
$$

Sendo assim, obtém-se que a incerteza combinada da densidade do óleo é dada por

$$
\left(\delta \rho_{0}\right)^{2}=\left(\frac{\rho_{w} \delta h_{w}}{h_{0}}\right)^{2}+\left(-\frac{h_{w} \rho_{w} \delta h_{0}}{h_{0}^{2}}\right)^{2}+\left(\frac{h_{w} \delta \rho_{w}}{h_{0}}\right)^{2} .
$$

Dividindo a Eq. (7) por $\rho_{0}$ obtém-se a relação 


$$
\left(\frac{\delta \rho_{0}}{\rho_{0}}\right)^{2}=\left(\frac{\delta h_{w}}{h_{w}}\right)^{2}+\left(\frac{\delta h_{0}}{h_{0}}\right)^{2}+\left(\frac{\delta \rho_{w}}{\rho_{w}}\right)^{2}
$$

que é a incerteza relativa de nossa medição. Uma análise mais apurada e realista da Eq. (8) nos subsidia na decisão do que fazer para tornar a medição da densidade do óleo mais exata, sem perder de vista o compromisso com o custo. A Eq. (8) pode ser simplificada assumindo que a densidade da água possui incerteza nula $\delta \rho_{w} \equiv 0$, já que é conhecida com exatidão bem maior que as outras variáveis envolvidas no problema, e que $\delta h_{w}=\delta h_{0}$ pois o mesmo instrumento de medição é utilizado para medir ambas as alturas das colunas de água e de óleo. A partir daí nota-se que existem duas maneiras de diminuir a incerteza relativa. A primeira é diminuir a incerteza associada às medições das alturas das colunas de líquido. Isso seria possível, por exemplo, substituindo a régua comum por uma régua (opção mais onerosa) que apresente incerteza (do tipo B) dez vezes menor. Essa opção deve ser descartada, nesse caso em especial, devido à interface óleo-água ter um volume de interpenetração considerável. Este fato anularia a vantagem experimental de utilizar tal régua e conseguir leituras com exatidão da ordem de décimos de milímetro.

Observe que as medições das alturas das colunas de líquido, $(7,7 \pm 0,1) \mathrm{cm}$ e $(8,1 \pm 0,1) \mathrm{cm}$, são diferentes, mas como o resultado depende da razão entre elas, seria muito melhor obtê-las com uma incerteza relativa menor. Em outras palavras, a diferença entre estas medições é da ordem de grandeza da incerteza associada. Seria interessante que a diferença entre estas medições fosse maior que a incerteza associada a cada uma delas.

A partir daí, observa-se que a medição obtida não tem um valor muito exato devido à dificuldade de se medir as alturas a partir da superfície que separa a água do óleo. A incerteza dessas medições não pode ser melhorada. Observe na seção seguinte uma modificação simples e que diminui em muito o custo do experimento (pois não precisaríamos comprar tubos de vidro em U com um comprimento muito maior que $10 \mathrm{~cm}$ ) e ainda facilita o nivelamento cuidadoso do tubo em U. Os resultados obtidos com o método do tubo em U tradicional são reportados na seção 4 .

\subsection{Medição da densidade do óleo utilizando um tubo flexível em $\mathrm{U}$ - uma mangueira transparente de $3 \mathrm{~m}$ de comprimento}

A Fig. 3 mostra uma modificação simples e muito eficaz que tem por objetivo reduzir muito a incerteza relativa envolvendo a medição da densidade do óleo utilizando um tubo em U. Neste experimento trocamos o tubo em $\mathrm{U}$ de vidro por uma mangueira transparente de $3 \mathrm{~m}$ de comprimento (que pode variar de acordo com a vontade do experimentador, dependendo de seu interesse em melhorar a medição) acomodada em um tripé com garras que mantém a mangueira dobrada e com seus ramos próximos um do outro. Dessa maneira, a medição das alturas acontece de forma muito menos complicada, facilitando o nivelamento e ainda com a utilização de uma régua milimetrada muito maior, tendo como resultado uma redução da incerteza relativa das medições das alturas de coluna de água e de óleo.

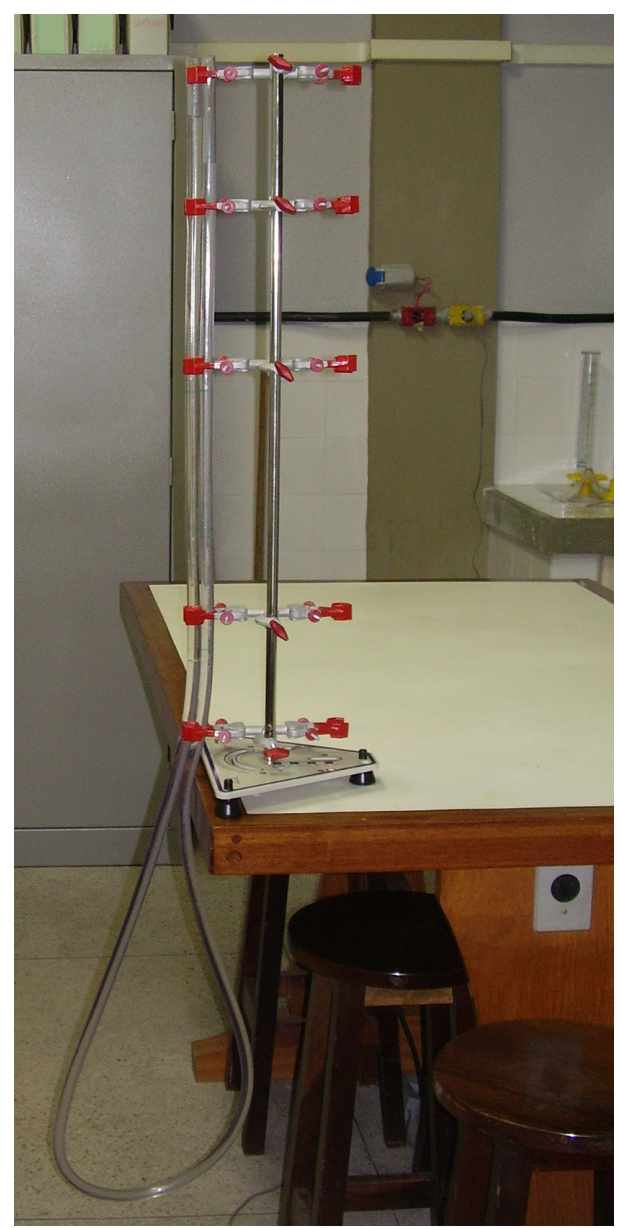

Figura 3 - Aparato experimental para a medição da densidade do óleo de cozinha utilizando uma mangueira transparente flexível como tubo em U.

A seqüência de fotos mostrada na Fig. 4 indica que agora a flutuação da medição devido a não uniformidade da superfície que separa a água do óleo não é tão crítica a ponto de modificar notavelmente o resultado da medição.

As medições são $(62,6 \pm 0,1) \mathrm{cm}$ e $(57,5 \pm 0,1) \mathrm{cm}$ para as alturas das colunas de óleo e água, respectivamente. A partir da Eq. (8) conclui-se que este aumento nas alturas das colunas de óleo e de água é muito desejável, visto que ambas as quantidades encontramse explicitadas no denominador. Os resultados obtidos com o método proposto neste trabalho são reportados na seção 4. 

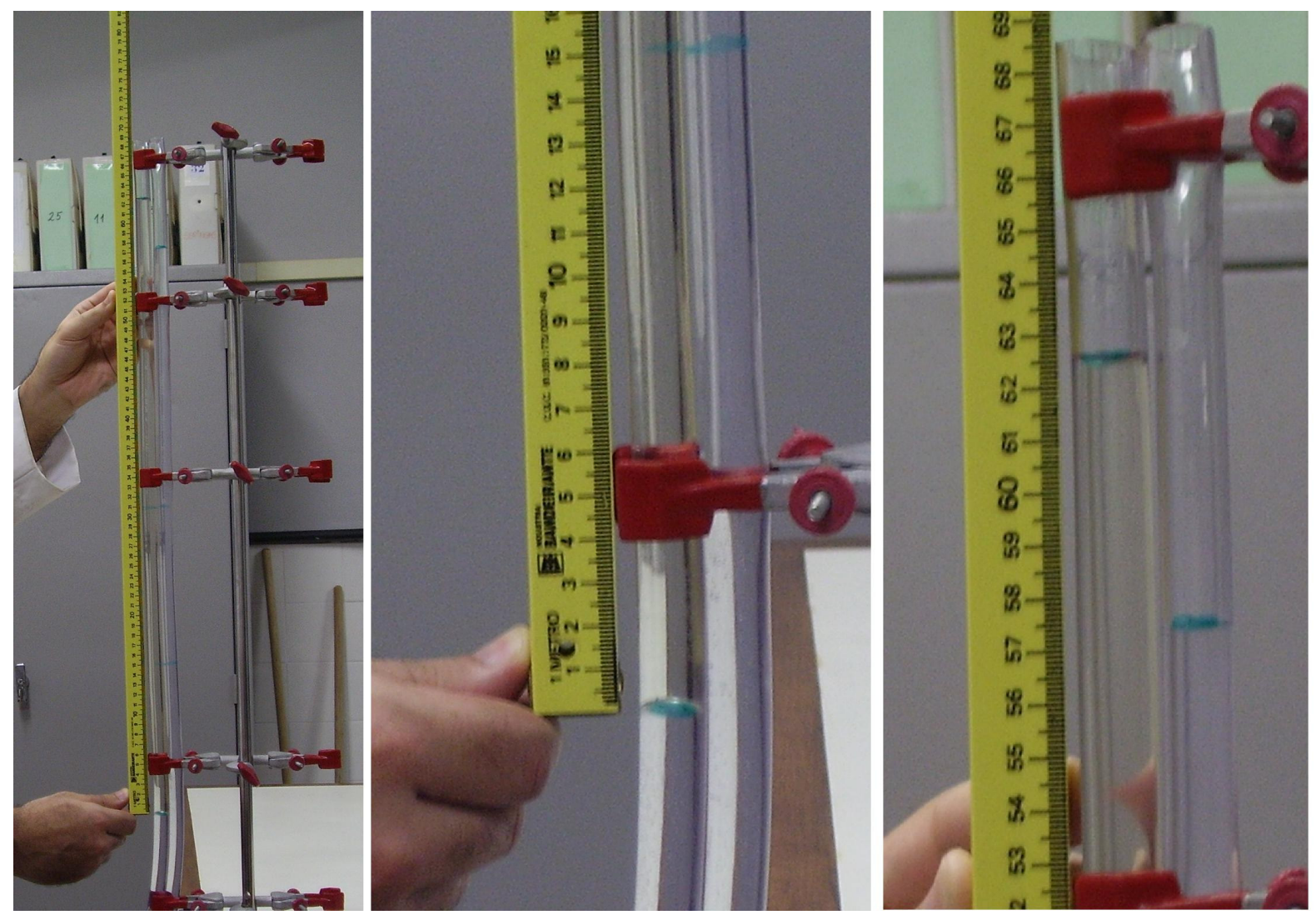

Figura 4 - Medição experimental da densidade do óleo de cozinha utilizando uma mangueira transparente flexível como tubo em U.

\section{O coeficiente de vantagem de um ex- perimento}

Em qualquer tipo de laboratório de física experimental existe um impasse entre a qualidade dos resultados obtidos e o custo que isso acarreta para a instituição mantenedora do mesmo. Até mesmo em laboratórios de simulação computacional, onde cálculos mais rápidos e acurados são cada vez mais necessários, deve-se pesar o quanto a compra de máquinas mais possantes e, conseqüentemente, mais rápidas, são realmente vantajosas. O gestor do ambiente de pesquisa pode adotar uma série de critérios para decidir como pesar os fatores exatidão e custo.

Tratando-se de experimentos de cunho didático, porém mantendo compromisso com a qualidade dos dados apresentados, é desejável que os mesmos apresentem incertezas relativas tão pequenas quanto possíveis, sem deixá-los demasiadamente onerosos. No presente trabalho, o um critério para escolha de um entre vários experimentos, ou configurações de experimentos, é proposto. Define-se aqui o fator de vantagem de um experimento como o produto da incerteza da grandeza física medida $(\delta)$ pelo seu custo $(C)$

$$
F_{v}=\delta \times C
$$

A Eq. (9) é definida de forma que os experimentos mais desejáveis (alta qualidade das medições e baixo custo) são aqueles que apresentam coeficientes de vantagem baixos. Na próxima seção são apresentados os resultados obtidos e são analisados seus respectivos coeficientes de vantagem.

\section{Resultados}

Na presente seção, são apresentados os resultados obtidos por diferentes métodos, e configurações, para a medição da densidade do óleo de cozinha e suas respectivas incertezas. De posse desses resultados o professor poderá avaliar a incerteza e o custo de um experimento proposto. A Tabela 1 mostra as diferentes configurações para os dois métodos tratados neste trabalho.

A partir das Eqs. (1) e (5) foram obtidos, respectivamente, a densidade do óleo de cozinha pelo método direto e pelo método do tubo em U. As Eqs. (3) e (7) fornecem suas respectivas incertezas associadas. O fator de vantagem do experimento é dado pela Eq. (9). A Tabela 2 resume os resultados obtidos para as diferentes configurações e métodos apresentados na Tabela 1. 
Tabela 1 - Métodos de determinação da densidade do óleo e custo aproximado. Os respectivos (volume máximo - menor divisão) da proveta e bureta são, respectivamente, $(500 \mathrm{~mL}-5 \mathrm{~mL})$ e $(50 \mathrm{~mL}-0,5 \mathrm{~mL})$.

\begin{tabular}{llr}
\hline Configuração & Descrição do experimento & Custo (R\$) \\
\hline 1 & Método direto: balança digital $(2 \mathrm{~kg}-0,1 \mathrm{~g})+$ proveta graduada & 750,00 \\
2 & Método direto: balança digital $(2 \mathrm{~kg}-0,01 \mathrm{~g})+$ proveta graduada & 1000,00 \\
3 & Método direto: balança digital $(2 \mathrm{~kg}-0,1 \mathrm{~g})+$ bureta & 800,00 \\
4 & Método direto: balança digital $(2 \mathrm{~kg}-0,01 \mathrm{~g})+$ bureta & 1050,00 \\
5 & Método do tubo em U comercial + régua metálica + nível & 120,00 \\
6 & Método do tubo em U flexível: mangueira transparente + régua metálica & 40,00 \\
\hline
\end{tabular}

Tabela 2 - Resultados obtidos para a densidade do óleo com sua respectiva incerteza e fator de vantagem.

\begin{tabular}{lcccc}
\hline Configuração & Incertezas & $\rho_{0}\left(\mathrm{~g} / \mathrm{cm}^{3}\right)$ & $\delta \rho_{0}\left(\mathrm{~g} / \mathrm{cm}^{3}\right)$ & Fator de vantagem \\
\hline 1 & $\delta m=0,1 \mathrm{~g}$ e $\delta V=3 \mathrm{~mL}$ & 0,9117 & 0,0057 & 4,3 \\
2 & $\delta m=0,01 \mathrm{~g}$ e $\delta V=3 \mathrm{~mL}$ & 0,9117 & 0,0057 & 5,7 \\
3 & $\delta m=0,1 \mathrm{~g}$ e $\delta V=0,3 \mathrm{~mL}$ & 0,9117 & 0,0061 & 6,9 \\
4 & $\delta m=0,01 \mathrm{~g}$ e $\delta V=0,3 \mathrm{~mL}$ & 0,9117 & 0,0057 & 2,0 \\
5 & $\delta h_{o}=\delta h_{w}=0,1 \mathrm{~cm}$ & 0,951 & 0,017 & 0,1 \\
6 & $\delta h_{o}=\delta h_{w}=0,1 \mathrm{~cm}$ & 0,9185 & 0,0022 & \\
\hline
\end{tabular}

A partir dos dados apresentados na Tabela 2, e baseado no fator de vantagem, conclui-se que a melhor configuração para se medir a densidade do óleo de cozinha em um laboratório didático, desde que se deseje um balanço entre exatidão e baixo custo, é a Configuração 6 , proposta neste artigo.

\section{Conclusões}

A abordagem experimental e do cálculo de incerteza relativa dada neste artigo tem por objetivo estimular os futuros professores de física do curso de licenciatura a analisar diferentes experimentos e configurações possíveis para a realização de medições e de interpretação dos resultados. O fator de vantagem proposto neste artigo (Eq. (9)), pode fornecer uma estimativa da real necessidade de se modificar ou comprar equipamentos para a realização de um determinado experimento.

Este exemplo simples de um experimento de vasos comunicantes e medição da densidade do óleo de cozinha pode ilustrar como uma análise da incerteza relativa pode levar a um olhar diferente e a confecção de experimentos mais simples e de baixo custo, fornecendo dados experimentais de qualidade.

\section{Agradecimentos}

Os autores gostariam de agradecer ao professor Sérgio Henrique Silva Junior, que leciona as disciplinas Metrologia Dimensional e Química em nossa instituição de ensino, por suas valiosas sugestões neste trabalho.

\section{Referências}

[1] V.C. Barbosa e A.M.S. Breitschaft, Revista Brasileira de Ensino de Física 28, 115 (2006)

[2] P.A. Tipler, Física (Ed. LTC, Rio de Janeiro, 2000), v. $1,5^{\mathrm{a}}$ ed.

[3] A. Mendes e P.P. Rosário, Metrologia e Incerteza de Medição (Ed. Epse, São Paulo, 2005).

[4] J.H. Vuolo, Fundamentos da Teoria dos Erros (Ed. Edgard Blücher, São Paulo, 1996), $2^{a}$ ed.

[5] J.H. Vuolo, Revista Brasileira de Ensino de Física 21, 350 (1999). 